\title{
Determination of titanium dioxide in cement by Microwave Digestion-VIS Spectrophotometry
}

\author{
Zheng-gen Huang ${ }^{1,2^{*}}$, Tao Wang ${ }^{2}$, Xiao-bin $\mathrm{Li}^{1}$, Bei-jia $\mathrm{Li}^{1}$ \\ 1. National Quality Supervision and Inspection Test Center of Architecture Ceramic, Leshan 614000. \\ 2. College of Materials and Chemistry \& Chemical Engineering, Chengdu University of Technology, Chengdu 610059.
}

\begin{abstract}
A new method to determine titanium dioxide in cement by Microwave Digestion-DAPM VIS Spectrophotometry which has many advantages such as simple operation, safe and fast, dissolve completely is presented in this paper. The determination conditions and working parameters of the microwave digestion instrument including the species and dosage of digestion reagent, wavelength, reaction time and the dosage of DAPM were optimized. The results show that samples were dissolved by HNO3-HF-H2O2, 395nm as the wavelength and $12 \mathrm{~mL}$ of DAPM. The method has been validated by determination of titanium dioxide in national standard reference materials of GBW03201, GBW03201c, GBW03207 and GBW03205. The results were in agreement with certified values and the RSD was $1.07 \%-4.52 \%$. The recovery ratio was ranging from $91.8 \%$ to $108.0 \%$ and the $|\triangle \operatorname{lgC}|$ was less than 0.018 . Comparison with the traditional digestion method that analytical cement samples of Sichuan, there is no significant difference between the two methods by Student's $t$ test. This new method provide a new way for quick and accurate to determine the content of titanium dioxide in cement.
\end{abstract}

\section{Introduction}

Cement is the basic product of national economic construction, and it is widely used in real estate, infrastructure and rural construction. At present, my country is the world's largest cement producer and consumer, and its total output accounts for more than half of the world's total. The various physical and chemical components and contents in cement are closely related to the quality of cement products and later construction applications [1]. The titanium dioxide present in cement is usually substituted by bauxite and clay raw materials. Titanium dioxide has an impact on the physical and chemical properties of cement. The improvement has been proven. For example, the decomposition temperature of calcium carbonate can be reduced within the range of 0.1 $1.0 \%$, and the strength of the product can be increased by up to $20 \%$ during use. Titanium dioxide can form a solid solution with cement clinker minerals, which can increase the maturity Material quality; but if the content is too much, titanium dioxide can react with $\mathrm{CaO}$ to produce perovskite without hydraulic properties and replace $\mathrm{SiO} 2$, which consumes $\mathrm{CaO}$ and affects the strength of cement. Therefore, the simple, fast and accurate inspection of titanium dioxide in cement has important economic and practical significance for the quality control of the production process and the production of high-quality and stable cement products.

Traditional detection methods for the content of titanium dioxide in silicate materials such as cement mainly use ICP-AES method [3], atomic absorption method, XRF method, etc. [4], but such equipment is expensive and has high cost and limited popularity. Traditional detection methods sample pretreatment methods mainly include wet decomposition, melting decomposition and sintering decomposition methods $[5,6]$. The wet decomposition method is simple and fast, but the ability to decompose the silicate sample is poor; the melting decomposition method decomposes the sample completely, but the temperature is too high and timeconsuming, the solvent erodes the vessel used for decomposition, and introduces a large amount of alkali metal salts; the temperature of the sintering method Not high, but need to add a lot of flux. Microwave digestion technology is a new digestion method that has developed rapidly in recent years. Its advantages are high efficiency, fast speed, simple operation, safety and reliability. The microwave directly releases energy to the sample, which greatly improves the digestion speed. It is now widely used In the fields of geology, environment, metallurgy, biology, food, etc. [7-13], there are relatively few reports on the direct use of microwave digestion of cement to determine the content of titanium dioxide at home and abroad. This article uses microwave digestion to quickly process cement samples, and spectrophotometric determination of titanium dioxide content. The results prove that the method is simple, fast, accurate and precise, and is suitable for rapid determination of titanium dioxide.

\footnotetext{
*Corresponding author's e-mail: 1szjs2102660@aliyun.com
} 


\section{Materials and methods}

\subsection{Reagents and instruments}

Titanium standard stock solution $1000 \mathrm{mg} / \mathrm{L}$ (National Nonferrous Metals and Electronic Materials Analysis Center), concentrated sulfuric acid, concentrated nitric acid, concentrated hydrochloric acid, ascorbic acid, hydrogen peroxide, diantipyrine methane, etc. are all analytically pure (Chengdu Kelong Chemical Co., Ltd. Company), ultra-pure water; GBW03201, GBW03201c, GBW03207 and GBW03205 Portland cement national standard materials (Institute of Geology, National Building Materials Industry Bureau)

Titanium working solution: take $5 \mathrm{~mL}$ standard solution, constant volume of $2 \% \mathrm{H} 2 \mathrm{SO} 4$ in a $100 \mathrm{~mL}$ volumetric flask;

Visible light spectrophotometer (Tianjin Prius Instrument Co., Ltd. 721 spectrophotometer), microwave digestion apparatus (Shanghai New Instrument Microwave Chemical Technology Co., Ltd. MDS-6), boxtype resistance furnace (Hunan Xiangtan Instrument Co., Ltd.), electric heating plate (Beijing Zhongxing Weiye
Instrument Co., Ltd.), analytical balance (Mettler-Toledo ME104E).

\subsection{Test method}

\subsubsection{Sample preparation method}

Accurately weigh $0.2 \mathrm{~g}$ (accurate to $0.0001 \mathrm{~g}$ ), dry the constant weight sample at $105^{\circ} \mathrm{C}$, and place it in a polytetrafluoroethylene digestion tank. Add a few drops of water to wet the sample. One group adds $6.0 \mathrm{mLHNO} 3+2 \mathrm{mLHF}$, and the second group $6.0 \mathrm{mLHNO} 3+2 \mathrm{mLHF}+1 \mathrm{mLH} 2 \mathrm{O} 2$, add $8 \mathrm{~mL} \mathrm{HNO} 3$ for the third group, $6 \mathrm{mLHCl}+2 \mathrm{mLHNO} 3$ for the fourth group and $6 \mathrm{~mL}$ HNO3-2mLH2SO4 for the fifth group. After soaking until the reaction is stable and the foam is basically eliminated, tighten the lid and put it in the microwave digestion apparatus, as shown in Table 1. Conditions are resolved. After the digestion is completed, drive the acid at $140^{\circ} \mathrm{C}$ in the electric heating plate until the smoke is exhausted, add water to a constant volume in a $100 \mathrm{~mL}$ volumetric flask, and add a blank test at the same time

Table1. Microwave digestion conditions

\begin{tabular}{ccccc}
\hline step & $\mathrm{P} / \mathrm{W}$ & $\mathrm{t} / \mathrm{min}$ & ${ }^{\circ} \mathrm{C}$ & $\mathrm{t} / \mathrm{min}$ \\
\hline 1 & 400 & 5 & 120 & 1 \\
2 & 800 & 5 & 160 & 4 \\
3 & 1000 & 5 & 200 & 15 \\
\hline
\end{tabular}

\subsubsection{Sample determination}

Pipette $0,0.2,0.5,1.0,2.0 \mathrm{~mL}$ of titanium working solution into a $50 \mathrm{~mL}$ volumetric flask, respectively, add 5 $\mathrm{mL}$ of $2 \%$ ascorbic acid solution, $10 \mathrm{~mL}(1+1)$ hydrochloric acid, and $12 \mathrm{~mL}$ of diantipyrine methane solution $(30 \mathrm{~g} / \mathrm{L})$. Dilute with water to the mark, shake well, and measure after standing for 20 minutes. The concentration of titanium in this standard working fluid series is $0,0.2,0.5,1.0,2.0 \mu \mathrm{g} / \mathrm{mL}$. Take the developed blank solution as a reference, use a $1 \mathrm{~cm}$ cuvette at a wavelength of $395 \mathrm{~nm}$, measure the absorbance of the standard working solution with a spectrophotometer and draw a standard curve. Pipette an appropriate amount of the liquid to another set of $50 \mathrm{~mL}$ volumetric flasks, add $5 \mathrm{~mL} 2 \%$ ascorbic acid solution, $10 \mathrm{~mL}(1+1)$ hydrochloric acid, $12 \mathrm{~mL}$ diantipyryl methane solution, dilute to the mark with water, shake well, After standing for 10 minutes, measure, check the concentration on the standard curve and calculate the content of titanium dioxide in the sample.

\section{Results and discussion}

\subsection{Measuring wavelength}

The test uses a 721 spectrophotometer. Titanium reacts with diantipyrine methane in a $1.2 \mathrm{~mol} / \mathrm{L} \mathrm{HCl}$ medium to form a yellow complex. Pipette $50.00 \mu \mathrm{g} / \mathrm{mL}$ titanium working solution $2.0 \mathrm{~mL}$ into Add $10 \mathrm{~mL} \mathrm{HCl}, 12 \mathrm{~mL}$ of ascorbic acid solution and diantipyrine methane solution to a $50 \mathrm{~mL}$ volumetric flask, develop color for 20 minutes, measure absorbance at different wavelengths (300$500 \mathrm{~nm}$ ), and the results are shown in Figure 1. 


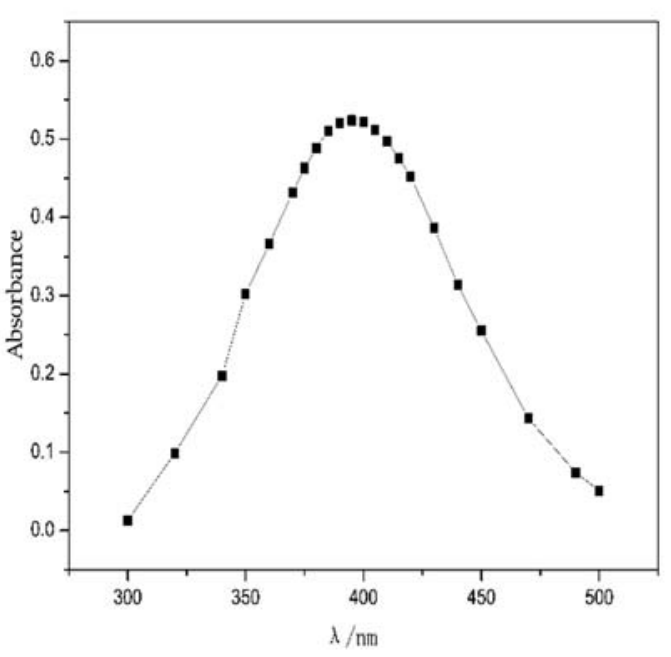

Fig 1. Absorption curve

It can be seen from the figure that in the hydrochloric acid medium, the maximum absorption wavelength of the complex formed by titanium and diantipyrylmethane is between $385-400 \mathrm{~nm}$. In order to obtain high sensitivity and reduce the test error, $395 \mathrm{~nm}$ is selected as the absorption wavelength for subsequent experiments.

\subsection{Drawing of working curve}

According to the test method in 1.2.2, draw a working curve of absorbance value (y) versus the solubility of titanium standard solution $(\mathrm{x}, \mu \mathrm{g} / \mathrm{mL})$, and the results are shown in Figure 2. The regression equation of the standard working curve is $y=0.26903 \mathrm{x}+0.00412$, which conforms to the Beer-Lambert law in the range of $0-2.0 \mu \mathrm{g} / \mathrm{mL}$, the absorbance A has a linear relationship with the standard

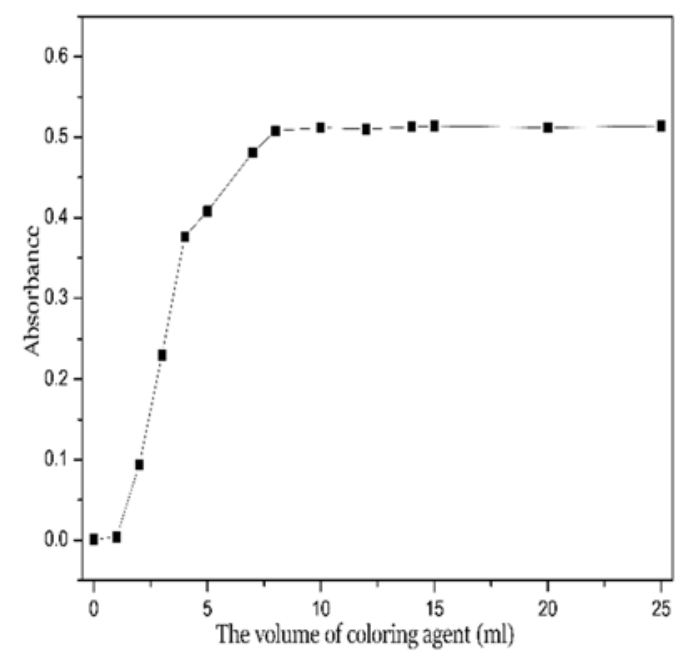

Fig 3. Effect of the amount of color developing solution

\subsection{Influence of color development time}

According to the test method of 1.2.2, the absorbance was measured at different color development times. The results

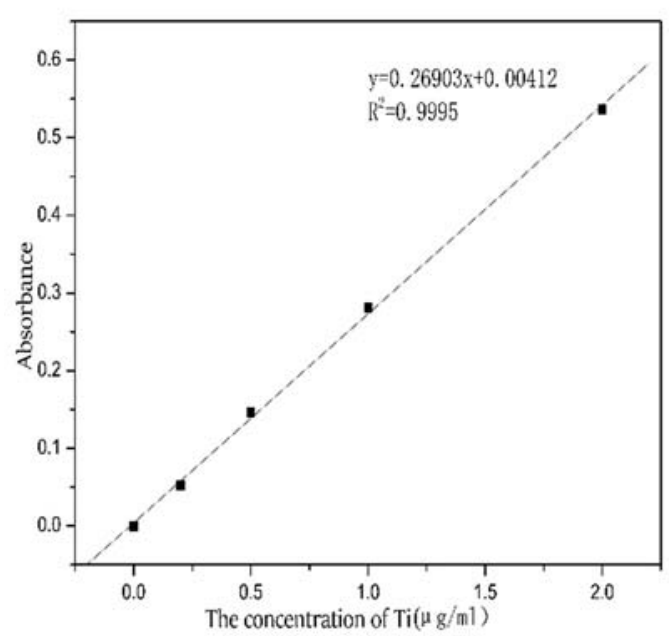

Fig 2. Working curve

solution, and the correlation coefficient R2 $=0.9995$.

\subsection{The effect of the amount of color developing agent diantipyrine methane}

Pipette an appropriate amount of $50 \mu \mathrm{g} / \mathrm{mL}$ titanium working solution, add $10 \mathrm{~mL} \mathrm{HCl}$, ascorbic acid solution, add different content of the color developing solution diantipyrine methane solution $(0-25 \mathrm{ml})$, develop color for 20 minutes, measure the absorbance, the results are shown in Figure 3. It can be seen from the figure that when the added color solution reaches the peak at about $10 \mathrm{~mL}$, the absorbance reaches the highest. In order to ensure that the color solution is sufficient to undergo complexation with all the titanium in it, the dosage of diantipyrylmethane is selected as $12 \mathrm{~mL}$.

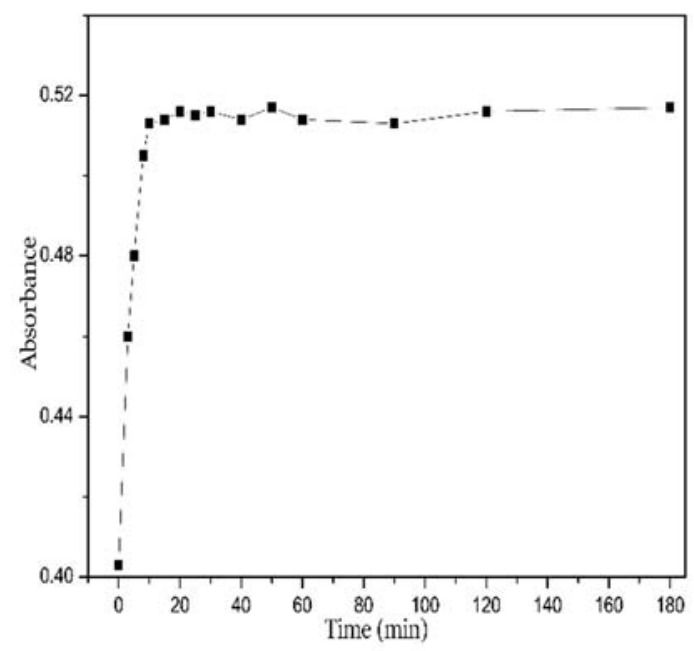

Fig 4. Effect of color development time

are shown in Figure 4. It can be seen from the figure that as the color development time increases, the absorbance gradually increases, and the color development of diantipyryl methane and titanium The absorbance over $15 \mathrm{~min}$ reaches the maximum and remains stable. In order 
to ensure that the color reaction is completed, the color development time is set to $20 \mathrm{~min}$.

\subsection{Selection of melting conditions}

In view of the characteristics of cement belonging to the silicate system, this paper selects 5 kinds of digestion systems, including HNO3-HF、HNO3-HF-H2O2、HNO3、 HNO3-H2SO4 and aqua regia. According to the conditions in Table 1, the four national standard materials of cement GBW03201, GBW03201c, GBW03207 and GBW03205 were subjected to microwave digestion test. The measurement results are shown in Table 2.

Table2. Comparison of determination results of titanium dioxide in different digestion systems

\begin{tabular}{ccccc}
\hline \multirow{2}{*}{ Digestion system } & $\begin{array}{c}\text { GBW03201 } \\
\text { value }(\mathrm{n}=4)\end{array}$ & $\begin{array}{c}\text { GBW03201c } \\
\text { value }(\mathrm{n}=4)\end{array}$ & $\begin{array}{c}\text { GBW03207 } \\
\text { value }(\mathrm{n}=4)\end{array}$ & $\begin{array}{c}\text { GBW03205 } \\
\text { value }(\mathrm{n}=4)\end{array}$ \\
\cline { 2 - 5 } & 0.25 & 0.20 & 0.48 & 0.36 \\
$\mathrm{HNO}_{3}-\mathrm{HF}$ & 0.26 & 0.21 & 0.50 & 0.38 \\
$\mathrm{HNO}_{3}-\mathrm{HF}_{-} \mathrm{H}_{2} \mathrm{O}_{2}$ & 0.23 & 0.16 & 0.45 & 0.33 \\
$\mathrm{HNO}_{3}$ & 0.24 & 0.19 & 0.49 & 0.35 \\
$\mathrm{HNO}_{3}-\mathrm{H}_{2} \mathrm{SO}_{4}$ & 0.25 & 0.19 & 0.48 & 0.36 \\
Aqua regia $_{\text {Recommended }}$ & 0.27 & 0.21 & 0.51 & 0.39 \\
value & &
\end{tabular}

It can be seen from the table that under the two digestion conditions of $\mathrm{HNO} 3$ and aqua regia, the sample still has a small amount of precipitation. Due to the high content of silicon in the cement and incomplete decomposition, the measured value is low; $\mathrm{HNO} 3-\mathrm{H} 2 \mathrm{SO} 4$ is also the recommended value of the standard material Some deviations may be due to the high boiling point of $\mathrm{H} 2 \mathrm{SO} 4$ and incomplete sample digestion. If the temperature of the microwave digestion apparatus is set too high and the pressure is too high, it is not advisable from the aspects of damage to the instrument and experimental safety; The added $\mathrm{H} 2 \mathrm{O} 2$ can effectively remove the organic matter in the sample. This paper chooses the HNO3-HF-H2O2 mixed acid decomposition system to dissolve ore.

\subsection{Accuracy and precision}

GBW03201, GBW03201c, GBW03207 and GBW03205 were tested in parallel six times to investigate the accuracy and precision of the method. The results are shown in Table 3. From the results in the table, it can be seen that the measured value of this method is basically consistent with the standard value. The absolute value of the logarithmic error $\triangle \lg C$ between the average and the standard value is within 0.018 , and the relative standard deviation (RSD) of the sample analysis is $1.07 \%-4.52 \%$, the method has high precision and repeatability, the absolute value of the relative error is less than $4.00 \%$, the method has good accuracy.

Table3. Method accuracy and precision

\begin{tabular}{|c|c|c|c|c|c|c|}
\hline \multirow{2}{*}{ Number } & \multicolumn{2}{|c|}{$\mathrm{W}_{\mathrm{TiO} 2} /(\%)$} & \multirow{2}{*}{$\begin{array}{l}\text { Recommended } \\
\text { value }(\%)\end{array}$} & \multirow{2}{*}{$\begin{array}{l}\text { Relative error } \\
(\%)\end{array}$} & \multirow{2}{*}{$\mathrm{RSD} \%$} & \multirow{2}{*}{$\triangle \lg C$} \\
\hline & $\begin{array}{l}\text { measured value } \\
\quad(n=6)\end{array}$ & $\begin{array}{l}\text { average } \\
\text { value }\end{array}$ & & & & \\
\hline GBW03201 & $\begin{array}{llll}0.26 & 0.27 & 0.25 \\
0.24 & 0.27 & 0.26\end{array}$ & 0.26 & 0.27 & -3.70 & 4.52 & -0.018 \\
\hline GBW03201c & $\begin{array}{lll}0.21 & 0.20 & 0.22 \\
0.21 & 0.20 & 0.20\end{array}$ & 0.21 & 0.21 & 0.00 & 3.95 & -0.016 \\
\hline GBW03207 & $\begin{array}{lll}0.50 & 0.50 & 0.52 \\
0.50 & 0.50 & 0.50\end{array}$ & 0.50 & 0.51 & -1.96 & 1.62 & -0.0036 \\
\hline GBW03205 & $\begin{array}{lll}0.38 & 0.38 & 0.39 \\
0.38 & 0.38 & 0.38\end{array}$ & 0.38 & 0.39 & -2.56 & 1.07 & -0.011 \\
\hline
\end{tabular}

\subsection{Method plus standard recovery test}

The ordinary Portland cement national standard materials GBW03201, GBW03201c, GBW03207 and GBW03205 were quickly dissolved by microwave digestion with HNO3-HF-H2O2 according to 1.2.1 sample pretreatment method. Each standard substance was treated with three solutions (each $0.2 \mathrm{~g}$ in a $100 \mathrm{ml}$ volumetric flask), one of which was used to measure the original background value, and the other two were added with an appropriate amount of titanium dioxide standard solution according to the gradient before dissolving the sample, according to 1.2. The test method of 2 develops color, determines the absorbance, calculates the titanium content according to the working curve, and then obtains the corresponding titanium dioxide content, and calculates the recovery rate. The results are shown in Table 4 . The results of the standard addition recovery test show that the standard addition recovery rate of the test sample is between 
91.8\%-108.0\%, indicating that the method has good accuracy.

Table4. Standard recovery test

\begin{tabular}{|c|c|c|c|c|c|c|c|}
\hline \multirow[t]{2}{*}{ Number } & \multicolumn{3}{|c|}{$\mathrm{W}_{\mathrm{TiO} 2} /(\mu \mathrm{g})$} & \multirow{2}{*}{$\begin{array}{c}\text { Recovery } \\
\text { rate } \%\end{array}$} & \multicolumn{2}{|c|}{$\mathrm{W}_{\mathrm{TiO} 2} /(\mu \mathrm{g})$} & \multirow{2}{*}{$\begin{array}{c}\text { Recovery } \\
\text { rate } \%\end{array}$} \\
\hline & $\begin{array}{l}\text { Background } \\
\text { value }\end{array}$ & Scalar & $\begin{array}{l}\text { measured } \\
\text { value }\end{array}$ & & Scalar & $\begin{array}{l}\text { measured } \\
\text { value }\end{array}$ & \\
\hline GBW03201 & 520.0 & 250.0 & 790.1 & 108.0 & 500.0 & 1030.2 & 102.0 \\
\hline GBW03201c & 420.0 & 250.0 & 660.2 & 96.1 & 500.0 & 956.2 & 107.2 \\
\hline GBW03207 & 1000.0 & 250.0 & 1232.4 & 93.0 & 500.0 & 1538.5 & 107.7 \\
\hline GBW03205 & 760.1 & 250.0 & 1026.2 & 106.4 & 500.0 & 1219.2 & 91.8 \\
\hline
\end{tabular}

\subsection{Sample analysis}

Use the established method-microwave digestion spectrophotometry to analyze the titanium dioxide content of cement samples produced in a certain place in Sichuan, and compare it with the national standard method GB/T176-2017[14] that uses conventional digestion, and use the t-test method[15] to determine the two Whether there are significant differences between these methods, the results are shown in Table 5. It can be seen from the table that the relative standard deviation RSD of the two methods of microwave digestion spectrophotometry and the national standard conventional digestion method to determine the content of titanium dioxide ( $\mu \mathrm{g} . \mathrm{g}-1)$ are less than $1.54 \%$ and $3.65 \%$, respectively, with good repeatability and Precision: The t-test method judges the two sets of data obtained by two methods for each sample. After calculation, all $\mathrm{t}<\mathrm{t} 0.05,6=2.45$ (confidence is $95 \%$, degree of freedom $f=6$ ) [15] It shows that there is no significant difference between the newly established microwave digestion method and the conventional digestion national standard method, and the measurement results of the two methods are in good agreement.

Table5. Comparison of the results of microwave digestion and conventional digestion methods

\begin{tabular}{|c|c|c|c|c|c|c|c|}
\hline \multirow{2}{*}{ Number } & \multicolumn{3}{|c|}{ Microwave digestion $W_{\mathrm{TiO}} /\left(\mu \mathrm{g} \cdot \mathrm{g}^{-1}\right)$} & \multicolumn{3}{|c|}{$\begin{array}{l}\text { National standard conventional digestion } \\
\text { methodW }_{\mathrm{TiO} 2} /\left(\mu \mathrm{g} \cdot \mathrm{g}^{-1}\right)\end{array}$} & \multirow{2}{*}{ t value } \\
\hline & $\begin{array}{l}\text { Sub-measured } \\
\text { value }\end{array}$ & $\begin{array}{c}\text { average } \\
\text { value }\end{array}$ & $\mathrm{RSD} \%$ & $\begin{array}{c}\text { Sub-measured } \\
\text { value }\end{array}$ & $\begin{array}{c}\text { average } \\
\text { value }\end{array}$ & RSD $\%$ & \\
\hline 2020LSTY001 & $\begin{array}{l}1034510287 \\
10321 \quad 10322\end{array}$ & 10318.8 & 0.23 & $\begin{array}{l}1023510487 \\
1042110122\end{array}$ & 10316.2 & 1.63 & 0.03 \\
\hline 2020LSTY002 & $\begin{array}{l}20932056 \\
21052047\end{array}$ & 2075.2 & 1.35 & $\begin{array}{l}21762089 \\
20542049\end{array}$ & 2092. 0 & 2.81 & 0.51 \\
\hline 2020LSTY003 & $\begin{array}{l}52345300 \\
52685200\end{array}$ & 5250.5 & 0.82 & $\begin{array}{l}51345323 \\
52345229\end{array}$ & 5230.0 & 1.48 & 0.46 \\
\hline 2020LSTY004 & $\begin{array}{l}45604457 \\
45654509\end{array}$ & 4522.8 & 1.12 & $\begin{array}{l}44564628 \\
46574530\end{array}$ & 4567.8 & 2.02 & 0.85 \\
\hline 2020LSTY005 & $\begin{array}{l}760746 \\
762770\end{array}$ & 759.5 & 1.31 & $\begin{array}{l}756780 \\
749768\end{array}$ & 763.2 & 1.79 & 0.44 \\
\hline 2020LSTY006 & $\begin{array}{l}562556 \\
560550\end{array}$ & 557.0 & 0.95 & $\begin{array}{l}545556 \\
550552\end{array}$ & 550.8 & 0.83 & 1.79 \\
\hline 2020LSTY007 & $\begin{array}{l}1520015300 \\
1566815200\end{array}$ & 15342.0 & 1.45 & $\begin{array}{l}1473415023 \\
1523415029\end{array}$ & 15005 & 1.37 & 2.22 \\
\hline
\end{tabular}




\begin{tabular}{lccccccc} 
2020LSTY008 & 102119987 & 10097.2 & 0.93 & 1086710278 & 10407.8 & 2.98 & 1.92 \\
& 1012310068 & & & 1018910297 & & & \\
2020 LSTY009 & 444459 & 451.8 & 1.54 & 430432 & 435.2 & 3.65 & 1.90 \\
& 448456 & & & 458421 & & & \\
2020 LSTY010 & 844829 & 829.2 & 1.31 & 810850 & 815.8 & 2.84 & 1.05 \\
& 818826 & & & 801802 & & & \\
\hline
\end{tabular}

\section{Conclusion}

Microwave digestion and dissolution technology is used to pre-treat ordinary Portland cement to provide work efficiency. The established method test results are accurate, fast, and precise. Compared with the traditional electric heating plate digestion method, the two methods have no significant difference. The established method provides a new way for the rapid and accurate detection of $\mathrm{TiO} 2$ in cement.

\section{References}

1. Dai Jinhui, Ge Zhaoming. The concept of inorganic non-metallic materials [M]. Harbin: Harbin Institute of Technology Press, 2004.

2. Xia Hui, Dang Xianmin, et al. Determination of titanium dioxide in wheat flour by microwave digestion-ultraviolet spectrophotometry[J]. Cereals and Food Industries, 2015, 22(6): 88-90.

3. Luo Haiying, Guo Xindong, Ye Jiarong, et al. Determination of titanium in food by microwave digestion-inductively coupled plasma atomic emission spectrometry[J]. Food Science, 2008, 29(3): 409-412.

4. Sun Baolian, Zhang Xiaoyan, Li Bo. Determination of trace titanium in alloy steel, copper alloy and aluminum alloy by graphite furnace atomic absorption method[J]. Rare Metal Materials and Engineering. 2003, 32(01): 76-79.

5. Wang Mohui, Lang Chunyan. Analysis of complex matter[M]. Chengdu: University of Electronic Science and Technology Press, 2002.

6. Compilation Group of Rock and Mineral Analysis. Rock and Mineral Analysis (Volume 1) [M]. Beijing: Geological Publishing House, 1991.

7. Li Zhiwei, Tai Zi'an, Ren Wenyan, Gao Zhijun, Li Yanhua. Determination of rare rare earth elements in black shale by microwave digestion inductively coupled plasma mass spectrometry[J]. Rock and Mineral Testing, 2010, 29(3): 259-262.

8. Yang Fucheng, Pei Wen. Microwave Digestion-Dual Channel Atomic Fluorescence Spectrometry Simultaneous Determination of Mercury and Arsenic in Blood[J]. Chinese Journal of Industrial Medicine, 2008, 21(3): 196-198.

9. $\mathrm{Ma} \mathrm{Li}, \mathrm{Si}$ Han. Simultaneous determination of heavy metal elements and rare earth elements in soil by microwave digestion of samples-inductively coupled plasma mass spectrometry[J]. Guide to Environmental Science. 2016, 35(02): 88-91.

10. Chen Yan, Nie Jihua, Zheng Shuanglai. Microwave digestion of diluted nitric acid assisted analysis of 5 elements in soil[J]. Chinese Journal of Health Inspection. 2016, 26(11): 1548-1551.

11. Baogui Rong, Zhao Yuying, Zheng Qingfu, Song Juanjuan, Zhou Hong. Simultaneous determination of 13 elements in tartary buckwheat leaves by microwave digestion-inductively coupled plasma atomic emission spectrometry[J]. Chinese Inorganic Analytical Chemistry. 2015, 5(04) : 88-90.

12. Zeng Y B, Xu H P, Liu H T, et al. Application of artificial neural networks in multifactor optimization of an on-line microwave FIA system for catalytic kinetic determination of Ruthenium ( III) [ J]. Talanta, 2001, 54( 4): 603-609.

13. Djingova R, Heidenreich H, Kovacheva P, et al . On the determination of platinum group elements in environmental materials by Inductively Coupled Plasma Mass Spectrometry and microwave digestion[ J]. Anal. Chim. Acta, 2003, 489( 2): 245251.

14. GB/T 176-2017 Cement chemical analysis method [S]

15. Wuhan University. Analytical Chemistry [M]. Beijing: Higher Education Press, 2006. 